\title{
Tecendo sobre o vestibular seriado: desafios e possibilidades
}

\author{
Weaving about the series entrance exam: challenges and possibilities \\ Tejiendo sobre el examen de ingreso a series: retos y posibilidades
}

Recebido: 14/11/2021 | Revisado: 19/11/2021 | Aceito: 20/11/2021 | Publicado: 22/11/2021

\author{
Jefferson Martins Costa \\ ORCID: https://orcid.org/0000-0002-3091-5012 \\ Instituto Federal de Minas Gerais, Brasil \\ E-mail: jefferson.martins@unesp.br \\ Danilo Calácio da Silva \\ ORCID: https://orcid.org/0000-0002-7133-8207 \\ Instituto Federal de Minas Gerais, Brasil \\ E-mail: danilocalacio@gmail.com \\ Niltom Vieira Junior \\ ORCID: https://orcid.org/0000-0003-1077-8302 \\ Instituto Federal de Minas Gerais, Brasil \\ E-mail: niltom.vieira@ifmg.edu.br
}

\begin{abstract}
Resumo
O Brasil possui diferenças significativas em relação às desigualdades educacionais. Somente $18,1 \%$ dos jovens cursam o ensino superior e a maioria dos estudantes que ingressam em um curso de graduação pertence a famílias de renda acima de 5 salários-mínimos. Mesmo com tendo políticas de cotas, percebemos que as desigualdades de acesso e permanência no ensino superior não foram superadas. O método de acesso ao ensino superior é reconhecido como um dos instrumentos que favorecem a desigualdade, na medida em que estudantes pobres e ricos são avaliados a partir dos mesmos critérios, mas possuem oportunidades de acesso ao conhecimento que são distintas e desiguais. O objetivo geral do presente estudo foi discutir os efeitos do vestibular seriado na democratização do ingresso ao ensino superior. Para tanto, foi realizada uma revisão de literatura. Os resultados indicam que métodos de vestibular seriado existem em algumas instituições de ensino superior no Brasil desde a década de 1980 e contribuem com o aumento das desigualdades socioeducacionais. Experiências de ingresso automático no ensino superior, como no caso da Argentina, contribuem com o aumento do número de estudantes de classes menos favorecidas ingressantes em cursos de graduação, embora não assegure a permanência. Conclui-se que a proposta de vestibular seriado efetiva um projeto de aumento das desigualdades e exclusão dos estudantes mais pobres do ensino superior.
\end{abstract}

Palavras-chave: Vestibular seriado; Ensino superior; Desigualdades.

\begin{abstract}
Brazil has significant differences regarding educational inequalities. Only $18.1 \%$ of young people attend higher education and the majority of students who enter an undergraduate course belong to families with income above 5 minimum wages. Even with quota policies, we realize that inequalities in access and permanence in higher education have not been overcome. The method of access to higher education is recognized as one of the instruments that favor inequality, as poor and rich students are evaluated based on the same criteria, but have different and unequal opportunities to access knowledge. The general objective of this study was to discuss the effects of the serial entrance exam in the democratization of admission to higher education. Therefore, a literature review was carried out. The results indicate that serial entrance exam methods exist in some higher education institutions in Brazil since the 1980s and contribute to the increase in socio-educational inequalities. Experiences of automatic entry into higher education, as in the case of Argentina, contribute to the increase in the number of students from lower classes entering undergraduate courses, although they do not guarantee permanence. It is concluded that the proposed serial entrance exam is effective in a project to increase inequalities and exclusion of the poorest students from higher education.
\end{abstract}

Keywords: Serial entrance exam; Higher education; Inequalities.

\section{Resumen}

Brasil tiene diferencias significativas en cuanto a desigualdades educativas. Solo el 18,1\% de los jóvenes asiste a la educación superior y la mayoría de los estudiantes que ingresan a un curso de pregrado pertenecen a familias con ingresos superiores a 5 salarios mínimos. Incluso con las políticas de cuotas, nos damos cuenta de que no se han superado las desigualdades en el acceso y la permanencia en la educación superior. El método de acceso a la educación superior es reconocido como uno de los instrumentos que favorecen la desigualdad, ya que los estudiantes pobres y ricos son evaluados con los mismos criterios, pero tienen diferentes y desiguales oportunidades de acceso al conocimiento. El objetivo general de este estudio fue discutir los efectos del examen de ingreso serial en la 
democratización de la admisión a la educación superior. Por tanto, se realizó una revisión de la literatura. Los resultados indican que existen métodos de exámenes de ingreso en serie en algunas instituciones de educación superior en Brasil desde la década de 1980 y contribuyen al aumento de las desigualdades socioeducativas. Las experiencias de ingreso automático a la educación superior, como en el caso de Argentina, contribuyen al aumento del número de estudiantes de clases bajas que ingresan a las carreras de grado, aunque no garantizan la permanencia. Se concluye que el examen de ingreso en serie propuesto es efectivo en un proyecto para aumentar las desigualdades y la exclusión de los estudiantes más pobres de la educación superior.

Palabras clave: Examen de ingreso seriado; Educación superior; Desigualdades.

\section{Introdução}

No Brasil, apenas $18,1 \%$ dos jovens cursam o ensino superior. Entre os alunos que cursaram o ensino médio em escolas pública, somente $36 \%$ ingressa em uma faculdade. Entre os egressos das escolas privadas, o percentual é de 79,2\% Instituto Brasileiro de Geografia e Estatística (IBGE). Tal contexto explicita a relação entre renda e educação que caracteriza o ensino superior no Brasil. De acordo com Helene (2013), geralmente apenas os estudantes que possuem acesso ao ensino médio de qualidade conseguem ingressar no ensino superior. Estudantes que possuem esse acesso são aqueles com maior renda familiar.

A desigualdade social existente no Brasil é, nesse contexto, reproduzida no acesso ao ensino superior. O processo de seleção para ingresso na universidade contribui para a exclusão de indivíduos das classes mais pobres do ensino superior. O principal meio de ingresso no ensino superior no Brasil é o Exame Nacional do Ensino Médio (ENEM).

Até no ano de 2009, o ENEM era utilizado como método de avaliação dos conhecimentos dos estudantes do ensino médio. A partir do final da década de 2000, o exame passou a ser utilizado para a seleção de estudantes para ingresso nas universidades. A nota do aluno passa a compor o Sistema de Seleção Unificada (Sisu). Alunos que não tenham nota para ingressar no curso pretendido em seu estado, podem utilizar a nota para tentar em regiões com menor concorrência ou optar por cursos para os quais as suas notas sejam suficientes para o ingresso. Para o governo, a ideia era reduzir as vagas excedentes em alguns cursos e estimular a mobilidade entre os alunos, formando profissionais para atuação em todas as regiões do país. No entanto, Silveira et al. (2015) discutem que o ENEM contribuiu com a maior segregação dos alunos, tendo em vista que os estudantes de regiões com menores índices de qualidade da educação passaram a concorrer com aqueles residentes nas capitais.

Os estudantes dos estados mais ricos ocupam o ranking entre aqueles que conseguem ingressar no ensino superior e os estudantes mais pobres não conseguiram obter a mobilidade pretendida pelo projeto, além de terem as vagas nas regiões de origem ocupadas por estudantes das cidades ricas. São Paulo, cidade com maior Produto Interno Bruto (PIB) do país, é a cidade que mais desloca estudantes para universidades localizadas nas regiões Norte e Nordeste. Além de concorrerem com estudantes que receberam uma melhor preparação para o exame, estudantes de cidades do interior em muitos casos não possuem recursos financeiros suficientes para realizar uma mudança para a capital, bem como custear moradia e alimentação (Silveira et al., 2015).

Nesse contexto, emerge o questionamento: quais as possibilidades de melhoria do acesso de estudantes de classes populares no ensino superior no Brasil?

O uso de um exame nacional único para a seleção de estudantes que desejam ingressar no ensino superior em um país caracterizado pelas desigualdades educacionais tanto em relação às regiões geográficas quanto às instituições escolares parece injusto e perverso.

Existe uma proposta do governo federal na qual as notas obtidas pelos alunos no Sistema de Avaliação da Educação Básica (Saeb) no decorrer do ensino médio, constituirão uma média de desempenho que poderá ser utilizada para ingresso no ensino superior.

O objetivo geral do presente estudo foi discutir os efeitos do vestibular seriado na democratização do ingresso ao ensino superior. Os objetivos específicos são identificar os tipos de vestibular seriado existentes, bem como os seus efeitos na democratização do acesso ao ensino superior e refletir sobre o vestibular seriado a partir de experiências internacionais. 


\section{Metodologia}

O artigo pautou-se por um estudo de caso utilizando a abordagem qualitativa e descritiva. Entendemos que este viés é o melhor caminho para atingirmos os resultados pretendidos, pois, corroboramos com Minayo (1994) ao afirmar que:

A metodologia pode ser compreendida como o caminho do pensamento e a prática exercida na abordagem da realidade. Portanto, embora seja uma prática teórica a pesquisa reúne pensamento e ação. Ou seja, nada pode ser intelectualmente um problema, se não tiver sido, em primeiro lugar, um problema da vida prática. As questões de investigação estão, portanto, relacionadas a interesses e circunstâncias socialmente condicionadas. (Minayo, 1994, p. 16 - 17).

De acordo com Lakatos e Marconi (2004, p. 269) “preocupa-se em analisar e interpretar aspectos mais profundos, descrevendo a complexidade do comportamento humano. Fornece análise mais detalhada sobre as investigações, hábitos, atitudes, tendências de comportamento etc". Na perspectiva dos campos teóricos estabelecidos para fundamentar a pesquisa terá uma abordagem qualitativa com aplicação de diferentes procedimentos metodológicos (Yin, 2010; Godoy, 2006; Roesch, 2009; Vianna, 2001). De acordo com Godoy (2006) a pesquisa qualitativa tem características que a tornam diferente do método quantitativo, são elas: 1) o ambiente natural como fonte direta de dados e o pesquisador como instrumento fundamental; 2) o caráter descritivo; 3) o significado que as pessoas dão às e à sua vida como preocupação do investigador; 4) enfoque indutivo.

De acordo com Falcão Vieira (2004, p. 15) o método qualitativo é "definido de acordo com o axioma de crenças do investigador. Tem importância fundamental à descrição detalhada do fenômeno e dos elementos que o envolvem, aos depoimentos dos atores sociais envolvidas, aos discursos, aos significados e aos contextos”. Compreende-se que a pesquisa qualitativa gera uma riqueza de dados, possibilita ainda a compreensão da totalidade de um fenômeno, explorando suas contradições e paradoxos.

Para sustentação teórica deste estudo utilizou-se uma pesquisa bibliográfica referente ao tema do estudo, pois, conforme Marconi e Lakatos (2011, p. 43) é necessário o “[...] levantamento de toda fonte bibliográfica já publicada, em forma de livros, revistas, publicações avulsas e imprensa escrita. Sua finalidade é colocar o pesquisador em contato direto com tudo aquilo que foi escrito sobre determinado assunto [...]". Para Moroz e Gianfaldoni (2006, p. 28) implica "na realização de um levantamento bibliográfico que consiste na seleção de obras que se revelem importantes e afins em relação ao que se quer conhecer”.

Deste modo, a pesquisa bibliográfica, nesse estudo, foi desenvolvida no catálogo da Biblioteca Digital Brasileira de Teses e Dissertações (BDTD), utilizando-se a ferramenta de busca avançada da plataforma com base nos descritores: Nesta pesquisa "Vestibular seriado" e "Vestibular", totalizando 30 trabalhos acadêmicos. Após a busca de dados, realizamos a leitura dos títulos, palavras-chave, resumos e selecionamos os trabalhos relacionados com a questão problema e objetivos da presente pesquisa. Portanto, foi necessário descartar algumas produções que não se articulavam com a temática do estudo.

A partir, então, da seleção das produções, realizamos novamente uma leitura seletiva, confirmando se os trabalhos selecionados tinham ou não uma relação ao tema pesquisado. Portanto, fizemos o reconhecimento do material bibliográfico e construímos uma síntese conforme veremos nos tópicos a seguir.

\section{O Sistema de Avaliação da Educação Básica E o Vestibular Seriado}

Schlichting et al (2004), explicam que o vestibular seriado está previsto no Parecer $\mathrm{n}^{\circ} \mathrm{CP}$ 98/99, que foi aprovado em 1999 e se refere a regulamentação dos processos seletivos para ingresso em instituições de ensino superior no Brasil (Brasil, 1999). De acordo, com o Parecer $n^{\circ} 98 / 99$ (1999, p. 2)

[...] o Art. 44 - A educação superior abrangerá os seguintes cursos e programas: a) II - de graduação, abertos a candidatos que tenham concluído o ensino médio ou equivalente e tenham sido classificados em processo seletivo. Art. 50 - As instituições de educação superior, quando da existência de vagas, abrirão matrícula nas disciplinas de seus 
cursos a alunos não regulares que demonstrarem capacidade de cursá-las com proveito, mediante processo seletivo prévio. Art. 51 - As instituições de educação superior credenciadas como universidades, ao deliberar sobre critérios e normas de seleção e admissão de estudantes, levarão em conta os efeitos desses critérios sobre a orientação do ensino médio, articulando-se com os órgãos normativos dos sistemas de ensino. Art. 50 - As instituições de educação superior, quando da existência de vagas, abrirão matrícula nas disciplinas de seus cursos a alunos não regulares que demonstrarem capacidade de cursá-las com proveito, mediante processo seletivo prévio. Art. 51 - As instituições de educação superior credenciadas como universidades, ao deliberar sobre critérios e normas de seleção e admissão de estudantes, levarão em conta os efeitos desses critérios sobre a orientação do ensino médio, articulando-se com os órgãos normativos dos sistemas de ensino.

A gênese do vestibular seriado no Brasil na década de 1980, quando pesquisadores da área de educação e o governo buscavam alternativas ao sistema de seleção de alunos para ingresso no ensino superior. Nesse contexto foram realizados Seminários denominados "Vestibular Hoje", sob responsabilidade do Ministério da Educação e de algumas universidades. Instituições como a Universidade de Brasília (UnB) propunham a realização de avaliações durante todo o ensino secundário, que permitissem uma análise mais abrangente do desempenho dos estudantes interessados em ingressar no ensino superior.

No início da década de 1990, foi concedida, pelo MEC, autorização para que a CESGRANRIO implementasse o Sistema de Avaliação Progressiva para Ingresso no Ensino Superior (SAPIENS). Na mesma década a UnB retomou as suas pesquisas a respeito do vestibular seriado, que se constituíram no Programa de Acesso ao Ensino Superior (PAS) iniciado em 1996. No mesmo sentido, a Universidade Federal de Santa Maria (UFSM) criou o Programa de Ingresso ao Ensino Superior (PEIES), que passou a ser modelo para outras instituições universitárias localizadas na região Sul do Brasil.

Coexistiram aos vestibulares seriados as propostas de avaliação em larga escala para a educação brasileira, que inclui o Exame Nacional de Cursos (ENC), o Exame Nacional do Ensino Médio (ENEM) e o Sistema Nacional de Avaliação da Educação Básica (SAEB).

A Prova Brasil e o Sistema de Avaliação da Educação Básica (Saeb) são avaliações para diagnóstico, realizadas em larga escala, criadas pelo MEC/INEP, com o objetivo de verificar a qualidade do ensino oferecido pelo sistema educacional brasileiro.

O Saeb é formado por duas avaliações complementares. A Avaliação Nacional da Educação Básica (Aneb) e a Avaliação Nacional do Rendimento Escolar (Anresc).

A Aneb utiliza como amostra os estudantes matriculados no $5^{\circ}$ e $9^{\circ}$ anos do país, em escolas de áreas urbanas e rurais e os estudantes de ensino médio que estão matriculados no $3^{\circ}$ ano. A Anresc é aplicada em alunos matriculados nos $5^{\circ}$ e $9^{\circ}$ anos do ensino fundamental público de redes estaduais, municipais e federais, em escolas urbanas e rurais com o mínimo de 20 alunos na série.

A Anresc é popularmente conhecida como Prova Brasil e tem os resultados segmentados por escola, cidade e estado. Os dados da Prova Brasil são utilizados também no cálculo do Índice de Desenvolvimento da Educação Básica (IDEB), calculado bienalmente.

As avaliações que compõem o Saeb subsidiam o IDEB, motivo pelo qual possuem a mesma periodicidade. Nelas são aplicadas provas de Matemática e Língua Portuguesa. Também são aplicados questionários socioeconômicos aos alunos participantes e à comunidade escolar.

A proposta de vestibular seriado implica incluir os $1^{\circ}$ e $2^{\circ}$ anos do ensino médio no Saeb. As notas registradas do $1^{\circ}$ ao $3^{\circ}$ ano comporão uma média que poderá ser utilizada como nota para ingresso na universidade. Os alunos poderão, ainda, optar por não utilizar a nota e realizar o ENEM da maneira tradicional. Para os alunos, o benefício estaria na substituição de uma avaliação que sintetiza todo o conhecimento adquirido em 3 anos de ensino médio por uma avaliação anual, correspondente a cada ano estudado.

No entanto, seria ingênuo acreditar que os efeitos do ENEM seriado serão sentidos somente pelos estudantes. Ao compor 
o Saeb, a avaliação passa a ser também uma avaliação da educação básica, o que incluir avaliar professores, currículos, metodologias de ensino e incluir as escolas em rankings que se tornarão anuais.

Em momentos que precedem as provas de avaliação em larga escala, professores, gestores e sistemas de ensino são pressionados para maximização dos resultados. Outro aspecto está relacionado à tradição do IDEB em explicitar os efeitos das desigualdades socioeconômicas no desempenho de alunos nas avaliações de larga escala (Soares \& Xavier, 2013). Sendo assim, estudantes de escolas públicas e com menor renda estariam entre aqueles que fatalmente terão as menores notas no vestibular seriado. Nesse sentido, verifica-se que o vestibular seriado não é eficiente na melhoria do acesso ao ensino superior. De fato, a proposta não parece ter esse objetivo. Contudo, em um momento no qual se propõe uma reformulação dos métodos de acesso ao ensino superior não seria razoável pensar em alternativas ao modelo vigente?

\title{
4. A Experiência Argentina
}

Na Argentina, o ensino superior está regulamentado na Ley 24.521 (Argentina, 2015). O ingresso ao ensino superior é gratuito e irrestrito. Não existem exames de admissão ou restrições.

\begin{abstract}
Atualmente, a oferta de ensino superior na Argentina compreende dois circuitos de formação paralelos e segmentados: a universidade (composta por universidades nacionais e provinciais financiadas com a contribuição do Estado Nacional, e universidades privadas) e a não universitária (terciária), predominantemente uma modalidade de formação docente, técnico-profissional e / ou mista entre as duas especialidades. Como a universidade, o ensino superior não universitário se desenvolve nos setores privado e público, neste último caso dependente das jurisdições, ou seja, das províncias e da Cidade Autônoma de Buenos Aires. Historicamente, a matrícula do nível universitário superior é notoriamente superior à do não universitário, visto que o primeiro circuito atinge maior legitimidade social. Nos últimos anos, o ensino universitário tem avançado na oferta e acreditação de ciclos de formação não universitários, razão pela qual o sistema de ensino superior se torna mais complexo sem ainda conseguir uma certa integração e articulação entre as duas modalidades (Suasnábar \& Rovelli, 2016, p. 83).
\end{abstract}

Os estudantes que desejam ingressar no ensino superior podem se inscrever em uma universidade pública e gratuita após a conclusão do ensino médio sem a necessidade de realização de alguma triagem. A formação para a docência no ensino primário é oferecida pelos Institutos Superiores de Formação Docente (ISFD), que oferecem formação em até 2 anos. O acesso gratuito e universal ao ensino superior foi instituído na Argentina a partir da década de 1960, pelos movimentos de oposição aos governos ditatoriais (Suasnábar \& Rovelli, 2016).

No entanto, críticos ao modelo argentino, corroborados por muitas pesquisas realizadas no Brasil, defendem que a universalização do acesso ao ensino superior na Argentina está associada a um maior percentual de evasão de estudantes no ensino superior, em consequência da ausência de um processo de seleção meritocrático, que escolha os alunos mais aptos para o ingresso e permanência. Tais pesquisas se baseiam na ideia de que os processos de seleção realizam uma triagem adequada dos estudantes que ingressarão no ensino superior, os quais possuem maiores habilidades para a formação científica (Vargas \& Heringer, 2017). Mas no Brasil, que adota um modelo de seleção rigoroso e excludente, há maior retenção de estudantes no ensino superior?

Vargas e Heringer (2017) explicam que o sistema educacional argentino apresenta os maiores índices de escolarização de todo o América Latina. Aproximadamente 37,6\% dos jovens entre 18 e 24 anos estão no ensino superior. No Brasil, esse índice é de 18,1\%. Destaca-se que na Argentina existe um número significativo de instituições superiores de nível técnico, as quais compõem o chamado ensino terciário e oferecem cursos para formação docente e outras atividades profissionais. Somadas às universidades, as matrículas das instituições técnicas de nível superior e das faculdades argentinas incluem 53,2\% de toda a população jovem entre 18 e 24 anos em algum curso de graduação.

No entanto, de acordo com o governo argentino, somente $29,7 \%$ dos estudantes concluem o curso no tempo previsto. 
Aproximadamente $48 \%$ dos estudantes argentinos abandona o curso ainda no primeiro ano de ingresso (Argentina, 2020). Para Rabossi (2013), o sistema educacional argentino, ao permitir o ingresso de todos os alunos no ensino superior, adota uma política permissiva, cujas consequências é um elevado percentual de evasão. Com base nesse argumento seria, então, de se esperar que no Brasil os dados sejam mais promissores.

De acordo com o Censo da Educação Superior realizado no Brasil no ano de 2017, os índices de evasão de estudantes no ensino superior no primeiro ano de ingresso eram de $49 \%$. Somente $42 \%$ dos estudantes que ingressam no ensino superior no Brasil conseguem concluir o curso (INEP, 2018). Tais dados não são distintos daqueles encontrados no país vizinho, que adota um modelo mais democrático de acesso à universidade. Mesmo que apresentem percentuais próximos, o percentual total da população argentina que consegue concluir o ensino superior se torna mais elevado do que no Brasil.

Nesse sentido, a facilitação do ingresso não pode ser considerada o motivo pelo qual as taxas de evasão são elevadas na Argentina, sobretudo em um contexto no qual o país viveu sucessivas crises econômicas que podem estar associadas às dificuldades encontradas pelos estudantes em permanecer no ensino superior, bem como em obter emprego após a conclusão do curso. Nenhum desses fatores, no entanto, está associado ao modelo de ingresso adotado no país. Do mesmo modo, o acesso universal não representa a inclusão de estudantes pobres no ensino superior. Em uma pesquisa comparativa entre os sistemas de acesso e permanência no ensino superior da Argentina, Chile e Brasil, foi identificado que todos os países possuem sistemas elitistas, mas o Brasil apresentou a maior desigualdade. Enquanto na Argentina, a taxa de estudantes pertencentes aos quantis mais pobres da população que ingressam no ensino superior foi de $21,6 \%$ e no Chile de $27,4 \%$, no Brasil esse percentual foi de 5,4\% (Vargas \& Heringer, 2017).

Embora as desigualdades sejam elevadas na Argentina, que adota o modelo de acesso universal, ela é significativamente maior no Brasil, onde os mecanismos de seleção são excludentes. No Brasil, os estudantes das famílias pertencentes aos quantis mais pobres nem sequer podem almejar o ingresso na universidade, tendo em vistas as dificuldades que encontrarão nos exames seletivos. A permanência desses estudantes no ensino superior dependerá de política de retenção. Trata-se de um tema a ser discutido no contexto argentino. No entanto, no Brasil as discussões ainda devem ser a respeito de como permitir que alunos pobres tenham acesso a oportunidade de ingressar em uma universidade.

\section{Eficácia do Vestibular Seriado no Acesso e Retenção de Estudantes no Brasil}

Borges e Carnielli (2005) realizaram uma análise dos efeitos do PAS da UnB na melhoria do acesso de indivíduos vindos das classes populares no ensino superior. Os autores realizaram uma aplicação de questionários para analisar as diferenças dos alunos ingressantes no curso de medicina pelo método do vestibular tradicional e do PAS, durante o ano de 2005. Os resultados indicaram que não foram identificadas distinções entre os ingressantes por meio do vestibular e por meio do PAS, no que se refere ao aspecto socioeconômico. Os autores verificaram, no entanto, que o PAS contribuiu com uma mudança no acesso dos alunos das classes mais abastadas, na medida em que eles tiveram uma facilitação do acesso por meio do processo seletivo, dado que ao adotar esse tipo de seleção, o aluno participava de um processo com menor concorrência.

No estudo de Borges e Carnielli (2005) foi verificado que 55\% dos alunos ingressantes nos cursos de Engenharia Civil, Medicina e Arquitetura cursaram pré-vestibular durante os três anos do ensino médio, na medida em que a criação do PAS também modificou o segmento de cursos preparatórios, os quais passaram a oferecer turmas com início já no primeiro ano do ensino médio. Os estudantes cujas famílias não possuem condições financeiras de custear tais cursos foram, nesse sentido, triplamente lesados, considerado cada um dos 3 anos de avaliação. Comparando os ingressantes por meio do PAS com aqueles que ingressaram por meio do vestibular tradicional, houve um aumento nas desigualdades sociais entre os estudantes. No curso de Engenharia, os ingressantes via PAS cujos pais possuem formação em nível superior corresponderam a 85,7\% e entre aqueles que ingressaram via vestibular foram $61 \%$. Trata-se de um indício de que os alunos ingressantes pelo PAS pertenciam 
majoritariamente a famílias de maior capital econômico e cultural, de modo que o referido método de seleção se constitui em um fortalecedor das desigualdades socioeducacionais, privilegiando o acesso de estudantes provenientes de famílias mais abastadas. O método de seleção foi ainda mais excludente sob o ponto de vista socioeconômico do que o vestibular tradicional (Borges \& Carnielli, 2005).

Considerando os três cursos, verifica-se que a renda familiar dos alunos que ingressam via PAS concentra-se entre a categoria de 11 a 15 salários-mínimos e a de mais de 40 salários-mínimos. Do total de alunos, 40,4\% com renda familiar acima de 40 salários-mínimos entraram por essa forma de acesso. Os alunos que entraram via vestibular apresentaram renda familiar entre a categoria de dois a cinco salários-mínimos e a de acima de 40 salários-mínimos. Dos alunos que ingressaram por essa via de acesso, 31,5\% concentram-se na categoria de renda familiar acima de 40 salários-mínimos (Borges \& Carnielli, 2005, p. 16).

Para além de um problema de formas de acesso ao ensino superior, existe um problema relacionado às desigualdades do sistema educacional brasileiro e de distribuição de renda. Os estudantes que participam dos processos seletivos para ingresso nas universidades brasileiras participam de provas para as quais grande parte não obteve acesso aos conhecimentos necessários para obter uma classificação adequada. As oportunidades são desiguais, mas os critérios de seleção são idênticos.

Os candidatos não concorrem em situação de igualdade. Há uma disputa entre desiguais por causa da estratificação social. A seletividade já se evidencia no ato da inscrição para o processo seletivo. Pelas razões apresentadas neste trabalho e em outros estudos, a formação educacional dos candidatos, em sua maioria, é determinada pela renda familiar (Borges \& Carnielli, 2005, p. 18).

O PAS foi considerado, nesse sentido, mais uma via de acesso ao ensino superior que privilegia as classes mais ricas, sobretudo em cursos cujas características dos ingressantes já correspondem ao perfil de famílias com maior renda.

Pires (2019) realizou uma análise da reprodução das desigualdades nas políticas públicas educacionais no Brasil. Ao analisar o PAS em comparação com o ENEM, o autor identificou que o primeiro processo seletivo tem uma tendência a selecionar os estudantes com maiores índices de renda, em comparação com o ENEM. Tal tendência foi reduzida durante os anos de 2014 a 2016, quando o sistema passou a admitir estudantes cotistas, mas ainda assim se manteve maior do que aquela identificada em outras formas de acesso.

\section{Considerações Finais}

A adoção da avaliação seriada como forma de ingresso no ensino superior brasileiro não é algo novo no debate público. Este tipo de avaliação já é utilizado em instituições com a UnB e a UFSM. No entanto, as pesquisas científicas a este respeito indicam que se trata de uma avaliação com características elitistas, que privilegiam os estudantes provenientes de famílias com melhores índices de renda e de educação formal.

Diante de tais dados, questiona-se os motivos pelos quais propostas nesse sentido retornam ao debate educacional brasileiro, como proposição do Ministério da Educação. Em um país com desigualdades educacionais e de renda significativas como o Brasil, estimular a construção de processo de seleção para ingresso no ensino superior que reforçam tais desigualdades parece um projeto político que fortalece a exclusão social. Em um contexto no qual os representantes do Ministério da Educação se posicionam a favor da redução do número de vagas no ensino superior, tal proposta não é ingênua.

A submissão de estudantes de classes sociais com menor renda a um processo seletivo realizado em 3 exames, durante o período de 3 anos, pode ter consequências na autoestima e na motivação desses estudantes para tentar outras formas de ingresso, como o ENEM. Ao obterem médias insuficientes nos exames anuais, estes alunos podem se sentir desmotivados em realizar outros tipos de avaliação. 
A avaliação seriada estimula, ainda, o aumento do mercado de cursos preparatórios, os quais contribuem ainda mais com as desigualdades entre aqueles que podem pagar e aqueles que não podem. Trata-se de um retrocesso quando o debate deveria se organizar em torno de possibilidades de universalização do acesso ao ensino superior, que conta com aproximadamente 2,8 milhões de vagas ociosas, de acordo com o Censo da Educação Superior.

Espera-se que os resultados deste trabalho contribuam para outros processos de reflexão sobre o tema pesquisa e, até mesmo, ajudem na construção de um projeto de reflexão, que busca as percepções docentes sobre o vestibular seriado versus vestibular tradicional. Ressalta-se ainda a importância de replicar estudos sobre esta temática nas escolas, visto que, o período de pandemia impossibilitou a realização in loco da pesquisa.

\section{Referências}

Argentina (2015). Síntesis de Información Estadísticas Universitarias 2018-2019. https://www.argentina.gob.ar/sites/default/files/sintesis_20182019_sistema_universitario_argentino_-_ver_final_1_0.pdf.

Borges, J. L. G. \& Carnielli, B. L. (2005). Educação e estratificação social no acesso à Universidade Pública. Cadernos de Pesquisa, 35(124), 113-139. https://www.scielo.br/j/cp/a/XHY7CN5QCTdxPDYRYMBZqkN/?format=pdf\&lang=pt

Godoy, C. K. \& Mattos, P. L. C. L (2006). Entrevista Qualitativa: instrumento de pesquisa e Evento Dialógico. In: Godoy, C. K., Bandeira-De-Mello, R. \& Silva, A. B. (org). Pesquisa Qualitativa em Estudos Organizacionais: Paradigmas, Estratégias e Métodos. Saraiva, $301-323$.

Helene, O (2013). Um Diagnóstico da Educação Brasileira e de seu Financiamento. Autores Associados.

INEP (2017). Censo da Educação Superior 2017 - Notas Estatísticas. https://download.inep.gov.br/educacao_superior/censo_superior/docu mentos/2018/censo_da_educacao_superior_2017-notas_estatisticas2.pdf.

Lakatos, E. M. \& Marconi, M. A. (2004). Metodologia científica. (4a ed.), Atlas.

Marconi, M. A. \& Lakatos, E. M. (2011). Metodologia do trabalho científico: procedimentos básicos, pesquisa bibliográfica, projeto e relatório publicações e trabalhos científicos. (7a ed.), Editora Atlas, p. 225.

Minayo, M. C. S. (1994). Pesquisa Social: teoria, método e criatividade. Vozes.

Moroz, M. \& Gianfaldoni, M. H. (2006) O processo de pesquisa: iniciação. Liber Editora.

Parecer CNE/CP n o 98/1999, aprovado em 6 de julho de 1999 - Regulamentação de Processo Seletivo para acesso a cursos de graduação de Universidades, Centros Universitários e Instituições Isoladas de Ensino Superior. https://www.google.com/search?q=sinonimo+de+na+medida+em+que\&rlz=1C1OKWM_ptBRBR971BR971\&oq=sinonimo+de+na+medida+em+que+\&aqs=chrome..69i57j69i64.8769j0j4\&sourceid=chrome\&ie=UTF-8.

Pires, R. R. (2019) org. Implementando Desigualdades: reprodução de desigualdades na implementação de políticas públicas. Ipea.

Rabossi, M. Universidade pública na Argentina: ineficiente e ineficaz? Ensino Superior Unicamp. https://www.revistaensinosuperior.gr.unicamp.br/int ernational-higher-education/universidade-publica-na-argentina-ineficiente-e-ineficazij.

Roesch, S. M. A. (2009). Projetos de Estágio e de pesquisa em Administração: guia para estágio, trabalhos de conclusão de curso, dissertações e estudos de casos. (3a ed.) Atlas.

Schlichting, A. M. S., Soares, D. H. P. \& Bianchetti, L. (2004). Vestibular seriado: análise de uma experiência em Santa Catarina. Psicologia \& Sociedade. 16(2), 114-126. https://doi.org/10.1590/S0102-71822004000200013. https://doi.org/10.1590/S0102-71822004000200013.

Silveira, F. L. da, Barbosa, M. C. B. \& Silva, R. da. (2015). Exame Nacional do Ensino Médio (ENEM): Uma análise crítica. Revista Brasileira de Ensino de Física 37(1).

Soares, J. F. \& Xavier, F. P. (2013). Pressupostos educacionais e estatísticos do Ideb. Educação \& Sociedade. 34(124), 903-923.

Suasnábar, C. \& Rovelli, L. I. (2016). Ampliaciones y desigualdades en el acceso y egreso de estudiantes a la Educación Superior en la Argentina. Pro-Posições 27(3), 81-104.

Vargas, H. \& Heringer, R. (2017). Políticas de permanência no ensino superior público em perspectiva comparada: Argentina, Brasil e Chile. Archivos Analíticos de Políticas Educativas, 25(72).

Vianna, I. de O. de A. (2001). Metodologia do Trabalho Científico: um enfoque didático da produção científica. E.P.U.

Falção Vieira, M. M. (2004). Por uma boa pesquisa (qualitativa) em Administração. In: Vieira, Falção \& Z., D. M. Pesquisa qualitativa em administração. FGV Editora.

Yin, R. (2010). Estudo de caso: planejamento e métodos. (4a ed.), Bookman, 